Pesq. Vet. Bras. 36(10):993-998, outubro 2016 DOI: 10.1590/S0100-736X2016001000012

\title{
Estudo epidemiológico e avaliação de fatores de risco da infecção por Toxoplasma gondii e achados clinico-patológicos da infecção aguda em cães admitidos em um Hospital Escola Veterinário ${ }^{1}$
}

\author{
Angelita D. Strital ${ }^{2}$, Michelle Igarashi ${ }^{3}{ }^{*}$, Livia S. Muraro², Daniel M. Aguiar ${ }^{4}$, \\ Thabata A. Pacheco ${ }^{4}$, João L. Garcia ${ }^{5}$, Silvio H. Freitas ${ }^{2}$ e Alexandre M. Amude ${ }^{2}$
}

\begin{abstract}
Strital A.D., Igarashi M., Muraro L.S., Aguiar D.M., Pacheco T.A., Garcia J.L., Freitas S.H. \& Amude A.M. 2016. [Epidemiological study and evaluation of risk factors for infection with Toxoplasma gondii and clinical and pathological findings of acute infection in dogs admitted at a Veterinary School Hospital.] Estudo epidemiológico e avaliação de fatores de risco da infecção por Toxoplasma gondii e achados clinico-patológicos da infecção aguda em cães admitidos em um Hospital Escola Veterinário. Pesquisa Veterinária Brasileira 36(10):993-998. Departamento de Ciências Básicas em Saúde, Faculdade de Medicina, Universidade Federal de Mato Grosso, Av. Fernando Corrêa da Costa 2367, Cuiabá, MT 78060-900, Brazil E-mail: michelle.igarashi@gmail.com

We aimed to verify the prevalence and risk factors for Toxoplasma gondii infection in dogs addimitted at the hospital. We also assessed the occurrence rates and the clinical and pathological repercussion of the acute infection by T. gondii in these animals. Antibodies were detected in 7\% (26/386) of a population of 386 dogs of both genders and different breeds and ages. Only variables, eating offal, rural origin and contact with cattle have significant values of $\mathrm{p}<0.05$. Dogs from rural areas showed higher risk $(\mathrm{OD}=7.00)$ of infection than those of urban origin. In 6.5\% (25/386) contact titles were detected (between 16 and 256); these titles do not necessarily mean acute infection, but only prior exposure. The recognition of prior infection by T. gondii is vital in those hospital patients. Depending on the cause of admission, although not being toxoplasmosis the responsible, the patient should receive prophylactic anti-parasite treatment or be monitored for further treatment in case of further acute occurrence of the disease by recrudescence of encysted bradyzoites. Only one dog $(3: 44 \%, 1 / 386)$ was admitted with high titer, what may be suggestive of acute infection (title of 4096). Although the dog with acute infection has shown neurological signs, caution is required not to extrapolate a false interpretation that toxoplasmosis is the main responsible for neurological signs, since numerous other cases included in this study had neurological signs without title of acute infection, even title of prior contact. Acute toxoplasmosis was not a significant clinic disease in this hospital; however differential diagnosis should be made in ill patients, especially those from rural areas, and definitive diagnosis must be reached for the correct therapeutic approach.
\end{abstract}

INDEX TERMS: Dogs, Toxoplasma gondii, toxoplasmosis, epidemiology, risk factors.

RESUMO.- Esse trabalho teve como objetivo estudar a prevalência e respectivos fatores de risco para infecção do Toxoplasma gondii em cães provenientes de uma população

\footnotetext{
${ }^{1}$ Recebido em 2 de setembro de 2015.

Aceito para publicação em 6 de Abril de 2016.

${ }^{2}$ Programa de Mestrado em Biociência Animal, Faculdade de Medicina Veterinária, Universidade de Cuiabá (Unic), Rua Manoel Fernandes Guimarães 3100, Cuiabá, MT 78065-900, Brasil.

${ }^{3}$ Departamento de Ciências Básicas em Saúde, Faculdade de Medicina, Universidade Federal de Mato Grosso (UFMT), Av. Fernando Corrêa
}

hospitalar. Além disso, avaliou-se as taxas de ocorrência e as repercussões clínico-patológicas da infecção aguda pelo T. gondii nesses animais. Anticorpos foram detectados em

da Costa 2367, Cuiabá, MT 78060-900. *Autor para correspondência: michelle.igarashi@gmail.com

${ }^{4}$ Laboratório de Virologia e Rickettsioses, Hospital Veterinário, Faculdade de Medicina Veterinária, UFMT, Av. Fernando Corrêa da Costa 2367, Cuiabá, MT 78060-900, Brasil.

${ }^{5}$ Departamento de Medicina Veterinária Preventiva, Faculdade de Medicina Veterinária, Universidade Estadual de Londrina (UEL), Rodovia Celso Garcia Cid, PR-445 Km 380 s/n, Campus Universitário, Londrina, PR 86057-970, Brasil. 
$7 \%(26 / 386)$ da população estudada, composta de 386 cães de ambos os sexos e diferentes raças e idades. Somente as variáveis, ingestão de vísceras, origem rural e contato com bovinos apresentaram valores significativos com $\mathrm{p}<0.05$. Adicionalmente os cães de origem rural apresentaram maiores risco (OD=7.00) de infecção do que aqueles de origem urbana. Em 6,5\% (25/386) foram detectados títulos de contato (entre 16 e 256); esses títulos não significam necessariamente infecção aguda e sim apenas exposição prévia. É de fundamental importância o reconhecimento da infecção prévia por $T$. gondii nesses pacientes hospitalares. Dependendo da causa da admissão, mesmo não sendo a toxoplasmose a responsável, o paciente deve receber o tratamento anti-protozoário profilaticamente ou ser monitorado para posterior tratamento em caso de reagudização da enfermidade por recrudescência dos bradizoítos encistados. Apenas um animal (3.44\%, 1/386) foi admitido com titulação elevada, o qual pode ser sugestivo de infecção aguda (titulo de 4096). Embora o animal com infecção aguda tenha sido apresentado com sinais neurológicos, cautela é necessária para não extrapolar uma falsa interpretação que a toxoplasmose é a grande responsável por quadros neurológico, uma vez que inúmeros outros casos incluídos nesse estudo tinham manifestações neurológicas e não tinham títulos de infecção aguda, nem mesmo título de contato prévio. A toxoplasmose aguda não foi uma afecção clínica expressiva nessa ambiência hospitalar, no entanto diagnóstico diferencial deve ser feito nos pacientes enfermos, principalmente os da área rural, e diagnostico definitivo deve ser alcançado para a correta conduta terapêutica.

TERMOS DE INDEXAÇÃO: Cães, Toxoplasma gondii, toxoplasmose, epidemiologia, fatores de risco.

\section{INTRODUÇÃO}

As infecções por protozoários são importantes alvos de estudos por conduzirem enfermidades com repercussões clínico-patológicas tanto para os animais quanto aos seres humanos (Dubey \& Beattie 1998). Nesse contexto a toxoplasmose se destaca por sua grande importância no cenário nacional tanto na saúde animal, quanto na saúde pública (Fioravanti 2012). Nos países em desenvolvimento, como o Brasil, a ocorrência da enfermidade é grande e isso pode ser justificada por uma série de fatores que contribuem para o processo de desenvolvimento e transmissão do parasita no meio ambiente como: grande contaminação ambiental, condições climáticas favoráveis, a manutenção de hospedeiros intermediários de vida livre, saneamento básico precário e ainda falta de leis efetivas que regem as responsabilidades e deveres dos proprietários e do Estado para com os animais de companhia (Dubey et al. 2006).

No Brasil a toxoplasmose ainda é um grande problema nacional e considerada como uma importante enfermidade negligenciada (Ferreira et al. 2011, Souza 2010, Fioravanti 2012). Embora existam muitos trabalhos epidemiológicos e estudos de fatores de risco da toxoplasmose no Brasil, a realização desse tipo de investigação e monitoramento no território nacional é importante, pois o território brasileiro é grande, com regiões bem distintas quanto a condições climáticas, culturais e socioeconômicas; logo, os estudos epidemiológicos e de fatores de risco para doenças infecto-parasitárias não podem ser extrapolados para todo o território brasileiro. Muitos aspectos epidemiológicos de enfermidades já bem estudadas e caracterizadas no centro-sul e sudeste ainda não são conhecidos em diferentes partes do território nacional.

Além das considerações acima, o estudo da epidemiologia e monitoramento da infecção por Toxoplasma gondii em animais tem grande importância na saúde pública, pois estes podem representar sentinelas da infecção para o ser humano. 0 íntimo contato dos cães com os seres humanos, assim como a ocupação dos ambientes, faz desses um bom sentinela para avaliar a contaminação ambiental e alimentar por uma série de agentes variando de toxinas e microorganismos (Assunção et al. 2005, Stucchi 2014). 0 diagnóstico da infecção de T. gondii nos cães indica um ambiente doméstico contaminado pelo parasito e isso representa um risco de infecção para a população humana, uma vez que tanto o homem quanto os cães estão expostos a um veículo de contaminação comum representado pelo ambiente e hábitos alimentares (Germano et al. 1985, Jackson et al. 1987, Varandas et al. 2001, Ullmann et al. 2008).

A toxoplasmose nos cães possui manifestações clínicas amplas que vão de quadros gastrentéricos, respiratórios, hemolinfáticos, oculares, musculares e/ou neurológicos (Dubey et al. 2006). Dessa forma, somente a detecção do agente ou do anticorpo anti-T. gondii em cães enfermos podem ajudar o clínico no diagnóstico definitivo da toxoplasmose (Dubey \& Lappin 2006, Bresciani et al. 2009). Segundo Varandas et al. (2001) e Ullmann et al. (2008), a detecção de anticorpos anti-T. gondii em cães, além de ser uma boa forma de diagnóstico definitivo da infecção, avalia a dispersão do protozoário no ambiente, demonstrando o papel do cão como animal sentinela para toxoplasmose e ressalta a importância do monitoramento nas ações de saúde pública para o controle desta zoonose (Varandas et al. 2001, Ullmann et al. 2008). Dessa forma, esse trabalho teve como objetivo estudar a prevalência de anticorpos anti- $T$. gondii e respectivos fatores de risco para infecção pelo $T$. gondii em cães, assim como avaliar as repercussões clínico-patológicas da infecção aguda em cães enfermos admitidos pela rotina do Hospital Escola Veterinário (HOVET) da Universidade de Cuiabá.

\section{MATERIAL E MÉTODOS}

Este estudo foi realizado prospectivamente entre o período de janeiro à dezembro 2013. A população de cães alvo do estudo foram animais admitidos no Setor de Clinica Médica de Animais de Companhia (CMPA) do Hospital Escola Veterinário (HOVET) da Faculdade de Medicina Veterinária da Universidade de Cuiabá (UNIC). 0 trabalho foi previamente avaliado pelo Comitê de Ética em Pesquisa da Universidade de Cuiabá com o registro $n^{\circ} 029$ CEP/UNIC - protocolo ${ }^{\circ}$ 2010-020.

Foi incluído no estudo somente animais provenientes da rotina hospitalar que realizaram o procedimento de venopunção para avaliação hematológica e/ou análise bioquímica clínica. 0 número da amostragem dos animais foi definido a partir da média de cães atendidos no Setor de CMPA semanalmente no Hospital Veteriná- 
rio da Universidade de Cuiabá (aproximadamente 25 atendimentos novos semanais). 0 programa Epi-Info 6.04 foi usado para o cálculo amostral com prevalência esperada de 50\%, precisão mínima de 10\% e intervalo de confiança (IC) de 95\% (Thrusfield 1995).

Foram coletadas amostras de sangue por venopunção cefálica ou jugular, após anti-sepsia prévia do local, com seringa $(10 \mathrm{ml}) \mathrm{e}$ agulha hipodérmica $(25 \times 0,70 \mathrm{~mm})$ descartáveis. 0 sangue obtido foi encaminhado ao laboratório de Análises Clínicas para avaliação hematológica e bioquímica; e após retração do coágulo o soro foi acondicionado em microtubo de polietileno de $1,5 \mathrm{~mL}$ e armazenado a $-20^{\circ} \mathrm{C}$ até o momento do processamento das amostras no Laboratório de Imunofluorescência da UNIC.

Após a coleta de sangue pela rotina hospitalar os proprietários foram convidados a participar da pesquisa e aqueles que aceitaram, responderam um questionário epidemiológico com informações sobre o animal e o ambiente, para análise dos fatores de risco. Foram analisadas variáveis como: origem dos cães (urbanos, rurais ou periurbanos), raça, idade, sexo, tipo de alimentação (comercial, caseira, carne crua), contato com felinos, ou outros hospedeiros intermediários (roedores), hábitos de caça e observação de sinais neurológicos.

A pesquisa de anticorpos anti-Toxoplasma gondii foi realizada por meio da técnica de Reação de Imunofluorescência Indireta (RIFI) a partir da cepa RH de T. gondii e segundo técnica descrita por Camargo (1974). Sucintamente, as amostras de soro foram utilizadas seguindo uma diluição inicial de 1:16 em solução salina tamponada (PBS) com a adição de 1\% de soroalbumina bovina. As mesmas foram adicionadas à lâmina previamente sensibilizada com taquizoítos de $T$. gondii e incubadas em câmara úmida a $37^{\circ} \mathrm{C}$ durante 30 minutos. Em todas as lâminas foram adicionados como controle soros caninos positivos (reativos) e negativos (não reativos). Depois de incubadas, as lâminas foram lavadas com tampão salina fosfato (PBS) ( $\mathrm{pH} 7.2$ ), seguido de um processo de secagem em temperatura ambiente. Posteriormente foi adicionado o conjugado anti-IgG canino marcado com isotiocianato de fluoresceína (Sigma ${ }^{\circledR}$ Diagnostics, St. Luis, Mo) e uma nova incubação foi feita seguida do mesmo procedimento de lavagem e secagem. Após secas totalmente, as lâminas foram montadas com glicerina tamponada e lamínula e a leitura foi realizada em microscópio de epifluorescência (Axio Scope Carl Zeiss Microscopy ${ }^{\circledR}$ ) na objetiva de 40x.

As reações foram consideradas positivas, quando os taquizoítos apresentavam fluorescência periférica total. Reações com fluorescência parcial ou apical foram interpretadas como negativas. Foram considerados positivos aqueles que reagiram em diluição a partir de 16; as amostras consideradas positivas passaram por sucessivas diluições na razão dois com objetivo de obter o título final. Títulos de 16, 32, 64, 128, 256 e 512 foram considerados títulos de contato (exposição prévia) e títulos iguais ou maiores que 1024 foram considerados títulos de infecção aguda (Zulpo et al. 2012).

As análises de associação foram realizadas pelos testes de Qui-quadrado ou Exato de Fisher quando necessário $(\mathrm{p}<0,05)$ para associar os resultados da sorologia com fatores de risco e variáveis analisadas.

\section{RESULTADOS}

Anticorpos anti-Toxoplasma gondii foram detectados em 26/386 (7\% 95\%IC, 4.5-9.8) da população estudada. Dos cães avaliados, $46 \%$ (12/26) dos animais foram admitidos com titulação de 16; 3.84\% (1/26) com 32; 19.23\% (5/26) com $64 ; 11.53 \%(3 / 26)$ com $128 ; 15.38 \%$ (4/26) com 256 ; e apenas um animal (3.44\%) foi apresentado com titula- ção de 4096. Dessa forma, dos 26 animais positivos para $T$. gondii, 25 apresentaram títulos de contato (menor ou igual a 512) e apenas um teve título de infecção aguda (maior ou igual a 1024). Dos animais com títulos de contato, cinco apresentaram febre, prostração e anorexia, e dois apresentaram perda de peso e vômito. Alterações como anemia, trombocitopenia, leucocitose, leucopenia e azotemia foram variações encontradas nos exames laboratoriais desses pacientes.

O animal com infecção aguda foi um macho, SRD, quatro anos de idade, origem urbana, tinha contato com roedores e gato, hábitos de caça, alimentação mista e apresentava como sinais clínicos febre, perda de peso, alterações dermatológicas e quadro neurológico multifocal caracterizado por convulsão e distúrbio espinhal torocolombar. Nos exames laboratoriais sanguíneos e bioquímicos foram encontrados 3,7 milhões de eritrócitos por dl, 8,9mg de hemoglobina por $\mathrm{dl}, 28 \%$ de volume globular, 60 mil plaquetas, 6,200 leucócitos totais (248 linfócitos, 5890 segmentados e 62 bastonetes), $8.8 \mathrm{~g} / \mathrm{dl}$ de proteínas totais, 26,1 de ALT e 0,8 de creatinina.

Do total de 386 cães analisados, $227(58,8 \%)$ eram fêmeas e $159(41,1 \%)$ machos; $102(26,4 \%)$ sem raça definida e $284(73,6 \%)$ com raça definida. Não houve associação entre as raças dos cães estudados e a presença de anticorpos anti-T. gondii; a maioria dos cães soropositivos foram animais de raça definida (Akita, Basset, Boxer, Chiuahua, Cocker, Dachshund, Fila, Labrador, Pinsher, Pitbull e Rottweiller). Dessas as raças, as mais representadas foram Pitbull e Rottweiller, com quatro cães positivos em cada uma das raças, porém sem significância estatística. Com relação a idade, $164(42,5 \%)$ foram menores que 36 meses ( 3 anos), 206 (53,3\%) superiores a 36 meses e em $16(4,1 \%)$ não foi possível determinar a idade (Quadro 1 ). Não houve diferença estatística entre cães positivos e faixa etária $\left(X^{2}=24,74\right.$ com $\left.\mathrm{p}=0,58\right)$, porém observou-se maior frequência de reações positivas em cães com idade avançada. Também não houve diferença estatística entre os sexos $\left(X^{2}=0.28\right.$ e $\left.\mathrm{p}=0,59\right)$.

Os resultados de frequência e a análise de associação das diferentes variáveis com a sorologia para T. gondii estão apresentados no Quadro 2. Distúrbios neurológicos foram observados em 12/386 animais (5,43\%; 95\% IC; 3,4-8,3), contudo em somente um animal foi verificado sorologia positiva $(3,84 \%)$. As variáveis significativas foram ingestão de vísceras $(14,47 \%$; 95\% IC; $11,2-18,4 ; \mathrm{OR}=3,5 ; \mathrm{P}<0.005)$, origem rural $(5,7 \%$; 95\%IC; 3,6-8,6; OR=7; $\mathrm{P}<0,005)$ e contato com bovinos (4,1; 95\%IC; 2,4-6.7; $\mathrm{OR}=5,2 ; \mathrm{P}<0,05)$.

\section{Quadro 1. Número e frequência de cães reagentes à Reação de Imunofluorescência Indireta para Toxoplasma gondii segundo faixa etária}

Faixa etária (meses) Número total de cães Número de cães positivos

\begin{tabular}{lcc}
\hline 0 a 12 meses & 86 & 3 \\
$>12 \mathrm{~m}$ a $48 \mathrm{~m}$ & 42 & 4 \\
$>24 \mathrm{~m}$ a $48 \mathrm{~m}$ & 61 & 5 \\
$>48 \mathrm{~m}$ a $72 \mathrm{~m}$ & 46 & 2 \\
$>72 \mathrm{~m}$ & 135 & 11 \\
Indeterminado & 16 & 1 \\
TOTAL & 386 & 26
\end{tabular}

Pesq. Vet. Bras. 36(10):993-998, outubro 2016 
Quadro 2. Frequência de anticorpos Anti-Toxoplasma gondii em cães hospitalares do município de Cuiabá/MT e análise de associação com as diferentes variáveis estudadas

\begin{tabular}{|c|c|c|c|c|c|}
\hline Fatores & Categoria & Cães positives n (\%) & Cães negativos n (\%) & OR (95\% IC) & $\mathrm{P}$ \\
\hline \multirow{2}{*}{ Acesso a rua } & Sim & $10(5,6)$ & $169(94,4)$ & 0,67 & 0,31 \\
\hline & Não & $19(8)$ & $214(92)$ & & \\
\hline \multirow[t]{2}{*}{ Hábitos de caça } & Sim & $7(6,7)$ & $97(93,3)$ & 0,94 & 0,88 \\
\hline & Não & $22(7)$ & $286(93)$ & & \\
\hline \multirow[t]{2}{*}{ Ingestão de carne crua } & Sim & $8(6,7)$ & $110(93,3)$ & 0,95 & 0,89 \\
\hline & Não & $21(7)$ & $273(93)$ & & \\
\hline \multirow[t]{2}{*}{ Ingestão de vísceras } & Sim & $9(34)$ & 47 (13) & 3,5 & 0,002 \\
\hline & Não & $17(65)$ & 313 (87) & & \\
\hline Alimentação caseira & & $1(10)$ & $9(90)$ & 1 & 0,13 \\
\hline Caseira+ração & & $15(9)$ & $231(91)$ & 0,51 & \\
\hline Ração & & $13(5)$ & $231(95)$ & 0,94 & \\
\hline Origem não informado & & 0 & $2(100)$ & $\mathrm{N} / \mathrm{Aa}$ & \\
\hline Peri-urbano & & 0 & $13(100)$ & $\mathrm{N} / \mathrm{A}$ & \\
\hline Rural & & $6(23)$ & $16(70)$ & 7 & 0,00001 \\
\hline Urbano & & $20(77)$ & 329 (91) & N/A & \\
\hline \multirow[t]{2}{*}{ Contato com outros cães } & Sim & $20(7)$ & $252(93)$ & 1,16 & 0,7 \\
\hline & Não & $9(6)$ & $131(94)$ & & \\
\hline \multirow[t]{2}{*}{ Contato com outros gatos } & Sim & $8(8)$ & $82(91)$ & 1,5 & 0,35 \\
\hline & Não & $18(6)$ & 278(93) & & \\
\hline \multirow[t]{2}{*}{ Contato com bovinos } & Sim & $4(25)$ & 12(75) & 5,2 & \\
\hline & Não & $25(6)$ & $371(94)$ & & 0,002 \\
\hline \multirow[t]{2}{*}{ Contato com roedores } & Sim & $8(30)$ & $110(30)$ & 1,01 & \\
\hline & Não & $18(69)$ & 250 (69) & & 0,84 \\
\hline
\end{tabular}

N/A = não avaliado.

\section{DISCUSSÃO}

No Brasil, estudos mostram que a prevalência de anticorpos anti-T. gondii em cães é heterogênea, com resultados variando $8,2 \%$ a $88,5 \%$ (Romanelli et al. 2007, Santos et al. 2009). Diferenças climáticas e culturais de cada região, tipo de população estudada e critério de inclusão pré-estabelecidos como sintomatologia dos animais avaliados, são exemplos de variáveis que podem gerar diversidades nos dados de prevalência em cada região do país. Lopes et al. (2011) e Zulpo et al. (2012) estudando amostras de cães hospitalares obtiveram prevalência de 50,89\% (57/112) em Londrina/PR e 18\% (100/530) no Piauí, respectivamente. Comparando com esses dados, o resultado de prevalência encontrado no presente estudo (7\%) pode ser considerado relativamente baixo. 0 município de Cuiabá, segundo o IBGE (Instituto Brasileiro de Geografia e Estatística), pode apresentar médias altas de temperaturas (acima de $40^{\circ} \mathrm{C}$ ) e médias baixas de umidade relativa do ar (abaixo de $20 \%$ ), fatores estes que poderiam contribuir para a não esporulação ou degradação de oocistos (Neves 2011) e assim menor contaminação ambiental.

Os sinais clínicos e alterações laboratoriais encontradas nos 25 caninos positivos com títulos de contato devem ser cautelosamente avaliados e não podem ser categoricamente assumidos como sendo causados pela infecção de Toxoplasma gondii; provavelmente as doenças de base responsável pela admissão hospitalar desses pacientes eram outras. No entanto, é de fundamental importância o reconhecimento da infecção prévia por T. gondii nesses pacientes hospitalares. Dependendo da causa da admissão, mesmo não sendo a toxoplasmose a responsável, o paciente deve receber o tratamento anti-protozoário profilaticamente ou ser monitorado para posterior tratamento em caso de reagudização da enfermidade por recrudescência dos bradizoítos encistados. Nos pacientes humanos previamente expostos e portadores de cistos teciduais de T. gondii, um grande fator de risco para a manifestação clínica e morte por toxoplasmose é a ocorrência de doença imunossupressora, como por exemplo, a AIDS (Lazo et al. 1998, Barsotti \& Moraes 2005). Em cães várias condições podem conduzir a imunossupressão como cinomose, leishmaniose, doenças endócrinas, e até mesmo gestação, entre outras. (Smith et al. 2007, Gemmill 2008). Além disso, em muitas outras enfermidades os protocolos terapêuticos utilizam drogas imunossupressoras como no lúpus, anemia hemolítica autoimune, pênfigo, tumores, e até alguns casos de encefalites (Smith et al. 2007, Coates \& Jeffery 2014, Nafe et al. 2014, Taylor et al. 2014).

Vários estudos têm avaliado a prevalência da toxoplasmose em pacientes portadores de enfermidades neurológicas (Plugger et al. 2011, Girardi et al. 2014), no entanto muito cuidado deve ser tomado na interpretação dos resultados; um resultado sorológico positivo não é o suficiente para incriminar o T. gondii como agente etiológico, uma vez que muitos animais possuem apenas títulos de contato e não de doença/infecção aguda. Plugger et al. (2011) realizaram uma pesquisa com 147 cães domiciliado/errantes que apresentavam sinais neurológicos como convulsão, paresia ou paralisia, ataxia, alterações de comportamento, alterações sensoriais somáticas, e encontrou uma prevalência de $21,08 \%$ para toxoplasmose, sendo os sinais neurológicos registrados nos pacientes positivos caracterizados por convulsões, ataxia e paralisia. Nesse estudo todos os pacientes exibiram títulos abaixo de 800, logo a extrapolação desses sinais neurológicos como sendo devidos a toxoplamose deve ser feito com respaldas. 
A toxoplasmose é tipicamente uma enfermidade multissistêmica (Dubey \& Lappin 1998). Os sinais de febre, perda de peso, sinais neurológicos, alterações eritrocitárias (anemia), leucocitárias (linfopenia) e plaquetárias (trombocitopenia moderada) encontradas no paciente com título de infecção aguda do presente trabalho, vêm de encontro com o conceito de acometimento multissistêmico na infecção pelo T. gondii. Existe a possibilidade de co-infecções com outros agentes como Ehrlichia canis (Girardi et al. 2014) e vírus da cinomose canina (CDV) (Aguiar et al. 2012); nessas situações mais de um agente poderia estar contribuindo com os sinais clínicos neurológicos/não-neurológicos, assim como as alterações laboratoriais (Giraldi et al. 2002). No entanto, a possibilidade de co-infecção com E. canis e/ ou CDV não foi avaliada nesse estudo.

Muita ênfase é dada às repercussões neurológicas nos pacientes caninos com toxoplasmose. Os autores acreditam que isso ocorra provavelmente pela transposição da importância da neurotoxoplasmose congênita humana. No entanto, no conhecimento dos autores, a ênfase que se tem dado a neurotoxoplasmose nos caninos em alguns trabalhos deve ser revista. No presente estudo a prevalência de toxoplasmose na população geral foi de $7 \%$ (26/386), enquanto na população com sinais neurológicos foi de 8,33\% $(1 / 12)$. Em um estudo anatomopatológico com cães portadores de doença neurológica que vieram a óbito, apenas dois de 60 animais tinham neurotoxoplasmose (Amude 2008). O conhecimento da prevalência da toxoplasmose, assim como outras enfermidades infecciosas causadoras de distúrbios neurológicos devem ser conhecidas nas respectivas regiões. Muitas outras enfermidades com potenciais neurotrópicos podem possuir taxas de prevalência superiores as registradas para toxoplasmose. Como exemplo, pode-se citar a erliquiose que pode chegar a uma prevalência de 70 a $90 \%$; em um estudo de fatores de risco em uma população hospitalar, a presença de sinais neurológicos foi considerado risco para infecção por $E$. $c a$ nis (Trapp et al. 2006). A cinomose tem sido reconhecida como a principal causa de óbito em animais (Silva et al. 2007) e a principal responsável por causar distúrbios neurológicos (Greene \& Appel 2006, Amude 2008). Portanto a neurotoxoplasmose nos caninos deve ser incluída no diagnóstico diferencial, entre os inúmeros agentes infecciosos que podem causar doença neurológica (Braund 1994, Vite 2005), como por exemplo, a erliquiose (Trapp et al. 2006), a leishmaniose (Grano et al. 2014) e a cinomose (Amude et al. 2007).

Em um estudo realizado em Cuiabá, utilizando uma amostra de cães com viés de seleção, onde se incluiu apenas animais com sinais neurológicos (58 cães), foi observado uma prevalência de $10,3 \%$ de anticorpos anti-T. gondii (Girardi et al. 2014). Se compararmos esse resultado com o resultado de prevalência (7\%) do presente estudo que avaliou uma população hospitalar geral, pode-se sugerir que na região de Cuiabá a prevalência de toxoplasmose em animais portadores de enfermidades neurológicas tende a ser similar a prevalência de toxoplasmose em animais de uma população hospitalar geral. Essa tendência também pode ser verificada no presente estudo quando comparamos os dados de prevalência desses dois tipos de população, uma vez que a prevalência de toxoplasmose na população geral $(7 \%, 26 / 386)$ foi similar a encontrada na população com sinais neurológicos $(8.33 \%, 1 / 12)$.

As associações entre a presença de anticorpos anti- $T$. gondii e as variáveis analisadas pelo teste do $\mathrm{X}^{2}$ ou Exato de Fisher identificaram as variáveis, ingestão de vísceras, origem rural e contato com bovinos como estatisticamente significativo $(\mathrm{p}<0.05)$. Adicionalmente os cães de origem rural apresentaram maiores risco $(\mathrm{OD}=7.00)$ de apresentarem a toxoplasmose do que aqueles de origem urbana. Provavelmente esse risco maior pode estar ligado ao fato desses animais terem mais chances de ingerirem vísceras pelos hábitos e costumes dos moradores rurais fornecerem aos cães as vísceras dos animais abatidos para o consumo humano. Corroborando com essa hipótese, Marques et al. (2009) constataram uma alta prevalência $(47,61 \%)$ em cães provenientes de 20 propriedades rurais no Estado do Mato Grosso do Sul. A significância encontrada no caso de contato com bovinos, não necessariamente ligam a toxoplasmose canina ao bovino, diferente do que acontece na neosporose. A significância matemática encontrada provavelmente está ligada ao fato de que cães que tem contato com bovino provavelmente moram em ambiente rural, e os riscos aumentados para esse tipo de ambiente.

Não houve diferença estatística entre cães positivos e faixa etária $\left(\mathrm{X}^{2}=24.74\right.$ com $\left.\mathrm{p}=0,58\right)$, porém observou-se maior frequência de reações positivas em cães com idade avançada, provavelmente devido a uma maior exposição ao agente. Esses dados estão em concordância com Garcia et al. (1999) que verificaram que a soropositividade dos cães aumenta com a idade.

\section{CONCLUSÕES}

Embora com prevalência menor do que em outras regiões do Brasil, as infecções por Toxoplasma gondii ocorrem em cães de Cuiabá/MT.

A baixa prevalência da infecção nos cães dessa região pode indicar um ambiente doméstico pouco contaminado pelo parasito com um risco menor de infecção para a população humana comparada com outras regiões brasileiras.

A infecção aguda não foi uma afecção clínica expressiva nessa ambiência hospitalar, no entanto diagnóstico diferencial deve ser feito nos pacientes enfermos, principalmente os da área rural, e diagnostico definitivo deve ser alcançado para a correta conduta terapêutica.

Agradecimentos.- Esse trabalho foi financiado pela Fundação de Amparo à Pesquisa do Estado de Mato Grosso (FAPEMAT).

\section{REFERÊNCIAS}

Aguiar D.M., Amude A.M., Dos Santos L.G.F., Ribeiro M.G., Ueno T.T.E.H., Megid J., Paes A.C., Alfieri A.F., Alfieri A.A. \& Gennari S.M. 2012. Canine distemper virus and Toxoplasma gondii co-infection in dogs with neurological signs. Arq. Bras. de Med. Vet. Zootec. 64:232-235.

Amude A.M. 2008. Encefalomielite pelo vírus da cinomose canina: aspectos neuroclínicos e neuropatológicos, e uso de técnicas moleculares para o diagnóstico seguro. Tese de Doutorado em Ciência Animal, Centro de Ciências Agrárias, Universidade Estadual de Londrina, Londrina, PA. 216p. 
Amude A.M., Alfieri A.A. \& Alfieri A.F. 2007. Clinicopathological findings in dogs with distemper encephalomyelitis presented without characteristic signs of the disease. Res. Vet. Sci. 82:416-422.

Assunção L., Pamplona M., Gentil K. \& Miranda D. 2005. Vigilância Epidemiológica: manual integrado de vigilância epidemiológica do botulismo, p.47-52. Secretária de Vigilância em Saúde, Ministério da Saúde,Brasília, DF. 88p.

Barsotti V. \& Moraes A.T. 2005. Neurotoxoplasmose como primeira manifestação da síndrome de imunodeficiência adquirida. Revta Fac. Ciênc. Méd., Sorocaba 7:20-22.

Braund K.G. 1994. Clinical Syndromes in Veterinary Neurology. 2nd ed. Mosby, St Louis. 477p.

Bresciani K.D.S., Souza A.P., Sartor A.A., Bellato V., Teixeira E.B., Pisetta G.M. \& Heusser Junior A. 2009. Ocorrência de anticorpos e fatores de risco para infecção por Toxoplasma gondii em cães, nas cidades de Lages e Balneário Camboriú, Santa Catarina, Brasil. Revta Bras. Parasitol. Vet. 18:52-56.

Camargo M.E. 1974. Introdução às técnicas de imunofluorescência. Revta Bras. Patol. Clín. 10:143-169.

Coates J.R. \& Jeffery N.D. 2014. Perspectives on meningoencephalomyelitis of unknown origin. Vet. Clin. North Am.,Sm. Anim. Pract. 44:1157-1185.

Dubey J.P. \& Beattie C.P. 1998. Toxoplasmosis in dogs (Canis familiaris), p.127-142. In: Ibid. (Eds), Toxoplamosis of Animals and Man. CRC Press, Boca Raton.

Dubey J.P., Chapman J.L., Rosenthal B.M., Mense M. \& Schueler R.L. 2006. Clinical Sarcocystis neurona, Sarcocystis canis, Toxoplasma gondii, and Neospora caninum infections in dogs. Vet. Parasitol. 137:36-49.

Dubey J.P. \& Lappin M.R. 1998. Toxoplasmosis and neosporosis, p.493503. In: Greene C.E. (Ed.), Infectious Diseases of the Dog and Cat. 2nd ed. W.B. Saunders Company, Philadelphia.

Dubey J.P. \& Lappin M.R. 2006. Toxoplasmosis and neosporosis, p.754775. In: Greene C.E. (Ed.), Infectious Diseases of the Dog and Cat. 3rd ed. Saunders Elsevier, St Louis, MO.

Ferreira I.M., Vidal J.E., Mattos C.C., Mattos L.C., Qu D., Su C. \& Pereira-Chioccola V.L. 2011. Toxoplasma gondii isolates: multilocus RFLP-PCR genotyping from human patients in São Paulo State, Brazil: identified distinct genotypes. Exp. Parasitol. 29:190-195.

Fioravanti C. 2012. Parasitas emergentes: formas brasileiras de toxoplasmose. Revtas Pesq. FAPESP 193:42-45.

Garcia J.L., Navarro I.T., Ogawa L. \& Oliveira R.C. 1999. Soroepidemiologia da toxoplasmose em cães e gatos de propriedades rurais do município de Jaguapitã, Estado do Paraná, Brasil. Ciência Rural 29:199-104.

Gemmill T.J. 2008. Self assessment: what is your diagnosis? J. Small Anim. Pract. 49:110-112.

Germano P.M.L., Erbolato E.B. \& Ishizuka M.M. 1985. Estudo sorológico da toxoplasmose canina, pele prova de imunofluorescência indireta, na cidade de Campinas. Revta Fac. Med. Vet. Zootec. USP 22(1):53-58.

Giraldi J.H., Bracarense A.P.F.R.L., Vidotto O., Tudury E.A., Navarro I.T. \& Batista T.N. 2002. Sorologia e histopatologia de Toxoplasma gondii e Neospora caninum em cães portadores de distúrbios neurológicos. Semina: Ciênc. Agrárias 23:19-14.

Girardi A.F., Lima S.R., Melo A.L.T., Boa Sorte E.C., Almeida A.B.P.F., Mendonça A.J., Aguiar D.M. \& Sousa V.R.F. 2014. Occurrence of anti-Toxoplasma gondii and Ehrlichia canis antibodies in dogs with nervous alterations assisted at a veterinary teaching hospital. Semina: Ciênc. Agrárias 5:1913-1922.

Grano F.G., Melo G.D., Belinchón-Lorenzo S., Gomez-Nieto L.C. \& Machado G.F. 2014. First detection of Leishmania infantum DNA within the brain of naturally infected dogs. Vet. Parasitol. 204:376-380.

Greene G.E. \& Appel M.J. 2006. Canine distemper, p.25-41. In: Greene G.E. (Ed.), Infectious Diseases of Dog and the Cat. 3rd ed. Saunders Elsevier, St Louis.

Jackson M.H., Hutchison W.M. \& Siim J.C. 1987. Prevalence of Toxoplasma gondii in meat animals, cats and dogs in central Scotland. Brit. Vet. J. 143:159-165.

Lazo J.E., Meneses A.C.O., Rocha A., Frenkel J.K., Marquez J.O., Chapadeiro
E. \& Lopes E.R. 1998. Meningoencefalites toxoplásmica e chagásica em pacientes com infecção pelo vírus da imunodeficiência humana: diagnóstico diferencial anatomopatológico e tomográfico. Revta Soc. Bras. Med. Trop. 31:163-171.

Lopes M.G., Mendonça I.L., Fortes K.P., Amaku M., Pena H.F.J. \& Gennari S.M. 2011. Presence of antibodies against Toxoplasma gondii, Neospora caninum and Leishmania infantum in dogs from Piauí. Revta Bras. Parasitol. Vet. 20:111-114.

Marques J.M., Isbrecht F.B., Lucas T.M., Guerra I.M.P., Dalmolin A., Silva R.C., Langoni H. \& Silva A.V. 2009. Detecção de anticorpos anti-toxoplasma gondii em animais de uma comunidade rural do Mato Grosso do Sul, Brasil. Semina: Ciênc. Agrárias 30:889-898.

Nafe L.A., Dodam J.R. \& Reinero C.R. 2014. In-vitro immunosuppression of canine T-lymphocyte-specific proliferation with dexamethasone, cyclosporine, and the active metabolites of azathioprine and leflunomide in a flow-cytometric assay. Can. J. Vet. Res. 78:168-175.

Neves D. 2011. Toxoplasma gondii, p.177-187. In: Kawazoe U. \& Mineo J.R. (Eds), Parasitologia Humana. 12 $2^{\mathrm{a}}$ ed. Atheneu, São Paulo. 546p.

Plugger N.F., Ferreira F.M., Richartz R.R.T.B., Siqueira A. \& Dittrich R.L. 2011. Occurrence of antibodies against Neospora caninum and/or Toxoplasma gondii in dogs with neurological signs. Revta Bras. Paratol. Vet. 3:202-206.

Romanelli P.R., Freire R.L., Vidotto O., Marana E.R., Ogawa L., De Paula V.S., Garcia J.L. \& Navarro I.T. 2007. Prevalence of Neospora caninum and Toxoplasma gondii in sheep and dogs from Guarapuava farms, Parana State, Brazil. Res. Vet. Sci. 82:202-207.

Santos T.R., Costa A.J., Toniollo G.H., Luvizotto M.C.R., Benetti A.H., Santos R.R., Matta D.H., Lopes W.D.Z., Oliveira J.A. \& Oliveira G.P. 2009. Prevalence of anti-Toxoplasma gondii antibodies in dairy cattle, dogs and humans from the Jauru micro-region, Mato Grosso state, Brazil. Vet. Parasitol. 161:324-326.

Silva M.C., Fighera R.A., Brum J.S., Graça D.L., Kommers G.D., Irigoyen L.F. \& Barros C.S.L. 2007. Aspectos clinicopatológicos de 620 casos neurológicos de cinomose em cães. Pesq. Vet. Bras. 27:215-220.

Smith P.M., Haughland S.P. \& Jeffery N.D. 2007. Brain abscess in a dog immunosuppressed using cyclosporine. Vet. J. 173:675-678.

Souza W. 2010. Doenças Negligenciadas. Academia Brasileira de Ciências, Rio de Janeiro. $56 \mathrm{p}$.

Stucchi C. 2014. Avaliação clínica-epidemiológica de botulismo em cães de uma população hospitalar do centro-oeste do brasil. Dissertação de Mestrado em Biociência Animal, Universidade de Cuiabá, Cuiabá, MT. 79p.

Taylor A., Talbot J., Bennet T.P., Martin P., Makara M. \& Barrs V.R. 2014. Disseminated Scedosporium prolificans infection in a Labrador retriever with immune mediated haemolytic anaemia. Med. Mycol. Case. Rep. 4:66-69.

Thrusfield M. 1995. Veterinary Epidemiology. 2nd ed. Blackwell, Oxford, 483p.

Trapp S.M., Dagnone A.S., Vidotto O., Freire R.L., Amude A.M. \& De Morais H.S. 2006. Seroepidemiology of canine babesiosis and ehrlichiosis in a hospital population. Vet. Parasitol. 10:223-230.

Ullmann L.S., Guimarães F.F., Fornazari F., Tomé R.O., Camossi L.G., Greca H., Silva R.C., Menozzi B.D. \& Langoni H. 2008. Ações de vigilância continuada, papel do cão como animal sentinela para toxoplasmose. Revta Bras. Parasitol. Vet. 17:345-347.

Varandas N.P., Rached P.A.A., Costa G.H.N., Souza L.M., Castagnoli K.C. \& Costa A.J. 2001. Frequência de anticorpos anti-Neospora caninum e contra Toxoplasma gondii em cães da região nordeste do Estado de São Paulo: correlação com neuropatias. Semina: Ciênc. Agrárias 22:105-111.

Vite C.H. 2005. Inflammatory diseases of the central nervous system. In: Vite C.H. (Ed.), Brand's Clinical Neurology in Small Animals: localization, diagnosis and treatment. International Veterinary Information Service (www.ivis.org), Ithaca, New York.

Zulpo D.L., Leite H.A.C., Cunha I.A.L., Barros L.D., Taroda A., Camargo Júnior V.E., Santos H.L.E.P.L. \& Garcia J.L. 2012. Ocorrência de anticorpos contra Leishmania spp., Neospora caninum e Toxoplasma gondii em soros de cães atendidos no Hospital Veterinário da Universidade Estadual de Londrina PR. Semina: Ciênc. Agrárias 33:1897-1906. 